\title{
Job Satisfaction and Sociodemographic Factors Affecting Garbage Workers' Quality of Life in Antakya (Antioch), Turkey
}

\author{
Nazan Savas ${ }^{1 *}$, Ersin Nazlican ${ }^{2}$ and Ebru Turhan ${ }^{1}$
}

${ }^{1}$ Department of Public Health, Mustafa Kemal University, Antakya, Hatay, Turkey

${ }^{2}$ Department of Public Health, Cukurova University, Adana, Turkey

\begin{abstract}
Objective: The aim was to evaluate job satisfaction and their sociodemographic characteristics effects on the quality of life $(\mathrm{QOL})$ of the garbage workers $(\mathrm{GW})$.

Methods: $185 \mathrm{GW}$ (94.4\% of total) were evaluated. They were given sociodemographic survey, scaler of job satisfaction (Job Descriptive Index (JDI)) and QOL Scale "WHOQOL-BREF-Turkish". Questions of JDI were compiled by researhers. Reliability and convenience of JDI were analysed. Correlation, student-T test and logistic regression were used.

Findings: $68.4 \%$ of job satisfaction had five contributing factors which are respectively management and overall job satisfaction, coworkers, pay, promotion and work itself. We found positive correlations $(p<0.05)$ between scorings of WHOQOL-BREF-Tr domains and JDI's components, except promotion. We also saw positive relations between WHOQOL-BREF-Tr scorings and some of workers' behaviors and habits ranging having particular hobbies, going to movies, visiting friends/family, and not having chronic disease $(p<0.01)$. Lack of satisfaction in each of 5 factors - dissatisfaction to management in all domains, dissatisfaction to work itself in social domain, dissatisfaction to coworkers in physical domain, dissatisfaction to pay in social and environmental domains caused a risk for poor QOL $(p<0.01)$. Not having a hobby also created a risk for $Q O L$ in social and environmental domains $(p<0.01)$.
\end{abstract}

Conclusion: The findings show that the QOL of the GW can be improved by improving the management, payment, and by encouraging and supporting them to have hobbies.

Keywords: Job satisfaction; Quality of life; Garbage workers

\section{Introduction}

Quality of life (QOL) can be defined as an individual's perception of his/her (person's) position in life in the context of the culture and value systems in which he/she (person) lives and in relation to his/ her (person) goals, expectations, standards and concerns. It is a broad ranging concept affected in a complex way by the person's physical health, psychological status, social relationships, and his/her (person) environment [1].

Among the most accepted definition of job satisfaction is by Locke who defines job satisfaction as a positive emotional feeling, a result of one's evaluation towards his job or his job experience by comparing between what he expects from his job and what he actually gets from it [2]. When he/she has feelings, he/she thinks about what he/she feels. Cognition and effects are thus inextricably linked, in his/her psychology and even in his/her biology. Thus, when evaluating his/ her jobs, as when he/she assesses anything most important to him/her, both thinking and feeling are involved [3]. Job satisfaction is the result of the interaction of the employees' values and his perception towards his job and environment [4].

Job satisfaction is affected by both individual factors and working conditions [5]. While individual factors are individual expectations, personality, work experience, sociability, working time and level of education, working conditions are difficulty level, payment, employment conditions, sociability of management, job security, promotion opportunities, equitable reward system and degree of involvement in decision making [5].

Several studies have suggested that there is a relation between working conditions and worker's QOL [6,7]. For example, Kudielka et al. [8] has suggested that high job demand, low job control and lack of social support at work exerts a significant impact on the self-reported health-related QOL. Also Heller et al. [9] study that was carried out on one hundred and fifty-three university employees working in a diverse set of occupations has shown the presence of both dispositional and environmental factors in job and life satisfaction. Besides Herzberg [10] has suggested, individuals have two levels of needs: the hygiene or maintenance needs (dissatisfiers) extrinsic to the job. Although both sets of factors operate to satisfy employee needs, the motivators provide the impetus for improved performance [10].

Municipal garbage workers usually work under un-hygienic conditions have many occupational risk factors. In this study, we sought to determine their job satisfactions and some sociodemographic factors affecting their QOL.

\section{Methods}

The study was performed in municipal garbage workers who work in the Antakya (centrum of Hatay) Municipality, in March 2010. Antakya is located in the southern part of Turkey on the Mediterranean coast and, its' population was 445,381 in 2009. Although Antakya Municipality has served to 202,216 persons at the same time [11]. The research group studied 198 municipal garbage workers. We aimed to

${ }^{*}$ Corresponding author: Nazan Savas, Department of Public Health, Mustafa Kemal University, Antakya, Hatay, Turkey, E-mail: drnazansavas@hotmail.com

Received November 08, 2011; Accepted January 31, 2012; Published February 02, 2012

Citation: Savas N, Nazlican E, Turhan E (2012) Job Satisfaction and Sociodemographic Factors Affectıng Garbage Workers' Quality of Lıfe in Antakya (Antioch), Turkey. Primary Health Care 2:110. doi:10.4172/2167-1079.1000110

Copyright: @ 2012 Savas N, et al. This is an open-access article distributed under the terms of the Creative Commons Attribution License, which permits unrestricted use, distribution, and reproduction in any medium, provided the original author and source are credited. 
interview all of them. Workers were informed about the study and their written consents were obtained, but two workers refused to participate at the study.

Workers were asked to fill in a questionnaire including three parts. First part included 18 questions related to their socio-demographic and job characteristics, second part, "The Job Descriptive Index" (JDI) had 26 questiones compiled by research group and third part - WHOQOLBREF- Turkish (TR), included 27 questions.

Approval from the Mustafa Kemal Univercity Medicine Faculty ethics committee was obtained to initiate the research. Additionally, sampled people were informed about the content of the research and their consents were taken. Data were obtained from the garbage workers by face-to-face interviewing.

\section{Job satisfaction index (JDI)}

The JDI is a scala that evaluates job satisfaction that was devoloped by Smith, Kendall, Lorne ve Hulin in 1969 [12]. It is the most widely used measurement of job satisfaction because of its careful construction and validation [13]. Turkish version of JDI was used and validated by Ergin [14]. The JDI that measures one's satisfaction in five facets (components): supervision, the work itself, pay, coworkers, promotions and promotion opportunities. Workers had to choose one of the 5 ratings meaning: $1=$ not very much; $2=$ not at all; $3=$ a little; 4 $=$ quite a lot; $5=$ a great deal.

In this research, questions were compiled by researhers. Five Facets had different number of questions respectively 5, 6, 5, 5 and 4 . Out of these, the last question was about general job satisfaction. First facet "Supervision" had one negative phrase question while second and fifth facets had two. Remaining questions in other facets had positive phrases.

\section{WHOQOL-BREF-TR}

WHOQOL-BREF is the abbreviated version of the original WHOQOL instrument. Although its long form includes 100 items, WHOQOL-BREF is a 26 item short version with five point Likert type response scale - generic QOL instrument. It was developed by WHO as a multilingual, multidimensional profile of QOL for crosscultural use $[15,16]$. WHOQOL are adapted by more than 40 cultures in the world. WHOQOL-BFEF has four broad domains namely: Physical, Psychological, Social Relations and Environmental. The instrument assesses satisfaction with life as well as the impact of disease or illness, and it captures positive and negative aspects of QOL. WHOQOL is a profile which has a good underlying theoretical conceptualisation of QOL [17]. It was validated in Turkish by Eser et al. [18]. It is a selfreported questionnaire containing four domains namely physical health (7 items), psychological status (6 items), social relationships (3 items) and environmental conditions ( 8 items). Turkish version had 27 questions last of which was related to Turkish background. Scoring in Environmental Area was named as CEVRE-TR. All items were scored on a Likert scale of 1 to $5(1=$ Very poor, $2=$ Poor, $3=$ Neither poor nor good, $4=$ Good, and $5=$ Very good; $1=$ Very dissatisfied, $2=$ Dissatisfied, $3=$ Neither satisfied nor dissatisfied, $4=$ Satisfied, and $5=$ Very satisfied; $1=$ Not at all, $2=$ A little, $3=$ A moderate amount, $4=$ Very much and $5=$ An extreme amount; $1=$ Not at all, $2=$ A little, $3=$ Moderately, $4=$ Mostly, $5=$ Completely; $1=$ Not at all, $2=$ A little, $3=$ A moderate amount, $4=$ Very much and $5=$ Extremely; or $1=$ Never, $2=$ Seldom, $3=$ Quite often, $4=$ Very often and $5=$ Always) [19]. The scores for some items were reversed to allow for comparison with other facets. The raw score of items within each domain was used to calculate the domain score by summing up the scores of the corresponding items in each domain. The domain score was converted to a transformed score (ranging from 4 to 20) to enable comparison to be made between domains consisting of unequal number of items. Domain scores were scaled in the positive direction, i.e. a higher score means a higher QOL [19].

\section{Statistical analysis}

Internal consistency of JDI was used for reliability analysis. Internal consistency of JDI was evaluated by Cronbach Alpha value. Ideally, Cronbach Alpha should be above 0.70 [20]. Validity analysis was performed using the construct and convergent validity. Construct validity was investigated by testing for principal components factor analysis. Varimax factor was used, screen plot was drawn, data whose eigen values are over 1 were taken into consideration. Convergent validity was determined by pearson correlation analysis.

Convenience of the data for factor analysis was realized with KaiserMayer-Olkin (KMO) and Bartlett test prior to factor analysis [21]. As the $\mathrm{KMO}$ value is higher than 0.60 , it indicates that it is convenient for factor analysis. Furthermore, Bartlett test is also an indicator of the convenience for factor analysis. Variance rate of first factor should be at least $30 \%$ of the total variance.

The correlations between scores of domains WHOQOL-BREFTR and scores of facets of JDI were analysed with pearson correlation. Besides the correlation between scores of domains WHOQOL-BREFTR and age, work duration of the job were analysed too. Relations between other sociodemographic factors and scores of WHOQOLBREF-TR domains were analysed by student ' $t$ ' test. Logistic Regression Analysis was completed between WHOQOL-BREF-TR domains and variables showing correlations and relations in order to find risk factors in workers' QOL. Variable scores that were received in WHOQOLBREF-TR domains were transformed to dicotom variables by dividing into two in median points. The statistical analysis was performed using SPSS for Windows, version 13; Chicago, IL.

\section{Results}

A total of 196 municipal garbage workers were included in this study, but $11(5.6 \%)$ workers were not included in the final analysis. Because they did not answer questions completely regarding JDI or WHOQOL. 91.4\% (169) of workers who answered completely (185) were garbage workers while $8.6 \%$ (16) were garbage truck drivers. Average employment time of a worker was $4.94 \pm 4.17$ years (range: 1-17 years). Distribution of number of workers based on working hours per day and timeframes respectively are; $74(40 \%)$ workers 07:00-17:00, 47 (25.4\%) workers 03:00-11:00, 47 (25.4\%) workers 22:00-06:00 and 17 (9.2\%) workers 11:00-21:00.

The mean age was $38.25 \pm 7.84$ years (range: 22-64 years) and the median age was 38 years. The mean number of their children was $2.39 \pm 1.39$ (range: $0-9$ children). Table 1 show that other sociodemographic factors of the study group.

\section{Results of reliability and construct validity test of JDI}

The KMO (Kaiser-Meyer-Olkin) value of JDI was measured as 0.858 and the Barlett's test value was 2185.53 ( $\mathrm{p}<0.05)$ (Table 2). Following items were excluded from the study due to their minimum effects. "It's hard to please my managers", "I have job security", "I have a respectful job”. Remaining 5 components, total of 23 items were 
Citation: Savas N, Nazlican E, Turhan E (2012) Job Satisfaction and Sociodemographic Factors Affectıng Garbage Workers' Quality of Lıfe in Antakya (Antioch), Turkey. Primary Health Care 2:110. doi:10.4172/2167-1079.1000110

Page 3 of 8

\begin{tabular}{|c|c|c|c|}
\hline Sociodemographic Factors & N (\%) & Sociodemographic Factors & N (\%) \\
\hline $\begin{array}{l}\text { The highest education } \\
\text { None at all } \\
\text { Primary school } \\
\text { Secondary school } \\
\text { Tertiary }\end{array}$ & $\begin{array}{l}6(3.2) \\
160(86.5) \\
18(9.7) \\
1(0.5)\end{array}$ & $\begin{array}{l}\text { Marital status } \\
\text { Single } \\
\text { Married } \\
\text { Living as married } \\
\text { Divorced } \\
\text { Widowed }\end{array}$ & $\begin{array}{l}6(3.2) \\
173(93.5) \\
1(0.5) \\
4(2.2) \\
1(0.5)\end{array}$ \\
\hline $\begin{array}{l}\text { Family type } \\
\text { Nuclear family } \\
\text { Large family } \\
\text { Broken family }\end{array}$ & $\begin{array}{l}157(84.9) \\
14(7.6) \\
5(2.7)\end{array}$ & $\begin{array}{l}\text { Occupation of wife } \\
\text { Housewife } \\
\text { Worker (insured) } \\
\text { Worker (uninsured) }\end{array}$ & $\begin{array}{l}166(89.7) \\
7(3.8) \\
12(6.5)\end{array}$ \\
\hline $\begin{array}{l}\text { Staying in house for rent } \\
\text { Absent } \\
\text { Present }\end{array}$ & $\begin{array}{l}73(39.5) \\
112(60.5)\end{array}$ & $\begin{array}{l}\text { Disabled/patient in the house } \\
\text { Absent } \\
\text { Present }\end{array}$ & $\begin{array}{l}171(92.4) \\
14(7.6)\end{array}$ \\
\hline $\begin{array}{l}\text { Currently ill } \\
\text { Absent } \\
\text { Present }\end{array}$ & $\begin{array}{l}159(81.1) \\
37(18.9)\end{array}$ & $\begin{array}{l}\text { Working children } \\
\text { Absent } \\
\text { Present }\end{array}$ & $\begin{array}{l}164(88.6) \\
21(11.4)\end{array}$ \\
\hline $\begin{array}{l}\text { Part time job } \\
\text { Yes } \\
\text { Sometimes } \\
\text { No }\end{array}$ & $\begin{array}{l}8(4.3) \\
24(13.0) \\
153(82.7)\end{array}$ & $\begin{array}{l}\text { Spending time with hobbies } \\
\text { Yes } \\
\text { Sometimes } \\
\text { No }\end{array}$ & $\begin{array}{l}47(25.4) \\
32(17.3) \\
106(57.3)\end{array}$ \\
\hline $\begin{array}{l}\text { Going out to movies/theaters/ball games } \\
\text { Yes } \\
\text { Sometimes } \\
\text { No }\end{array}$ & $\begin{array}{l}21(11.3) \\
39(21.1) \\
125(67.6)\end{array}$ & $\begin{array}{l}\text { Visiting friends/relatives } \\
\text { Yes } \\
\text { Sometimes } \\
\text { No }\end{array}$ & $\begin{array}{l}102(55.1) \\
58(31.4) \\
25(13.5)\end{array}$ \\
\hline
\end{tabular}

Table 1: Sociodemographic factors of garbage workers $(\mathrm{N}=185)$.

\begin{tabular}{|c|c|c|c|c|c|}
\hline Factors & 1 & 2 & 3 & 4 & 5 \\
\hline $\begin{array}{l}\text { Rate of variance explanained }(\%) \\
\text { Initial Eigenvalues (\% of variance) } \\
\text { Rotation sums of squared loading (\% of variance) }\end{array}$ & $\begin{array}{l}30.91 \\
16.22 \\
\end{array}$ & $\begin{array}{l}14.16 \\
15.24\end{array}$ & $\begin{array}{l}8.10 \\
14.97\end{array}$ & $\begin{array}{l}6.40 \\
10.88\end{array}$ & $\begin{array}{l}5.60 \\
7.98\end{array}$ \\
\hline \multicolumn{6}{|l|}{ Sentences (question number) } \\
\hline My manager is respectful and fair to me (1) & 0.807 & & & & \\
\hline My manager appreciates my work (3) & 0.800 & & & & \\
\hline I get support and help from my manager (2) & 0.787 & & & & \\
\hline My manager oversees my work (5) & 0.765 & & & & \\
\hline I am satisfied at my work in general (26) & 0.597 & & & & \\
\hline I have a responsibility at work (7) & 0.522 & & & & \\
\hline I get help from coworkers if I need it (20) & & 0.823 & & & \\
\hline I get along with my coworkers (19) & & 0.799 & & & \\
\hline My coworkers are hard working (18) & & 0.795 & & & \\
\hline My coworkers have responsibilities (17) & & 0.777 & & & \\
\hline I am not in a competition with my coworkers (21) & & 0.643 & & & \\
\hline Pay raise is more likely at my work (15) & & & 0.874 & & \\
\hline My pay is enough comparing to what else I can do (16) & & & 0.801 & & \\
\hline My pay is sufficient to my family needs (12) & & & 0.795 & & \\
\hline I get paid as much as what I contribute to my work (13) & & & 0.761 & & \\
\hline I get paid monthly and regularly (14) & & & 0.539 & & \\
\hline Promotion requires personal skills at my work (24) & & & & 0.787 & \\
\hline Unfair promotions happen at my work (23) & & & & 0.753 & \\
\hline I have very limited promotion opportunities (22) & & & & 0.664 & \\
\hline Promotions happen regularly at my work (25) & & & & 0.640 & \\
\hline My work is tiring (10) & & & & & 0.830 \\
\hline I have no insurance (9) & & & & & 0.673 \\
\hline I have appropriate equipment at my work (11) & & & & & 0.606 \\
\hline KMO (Kaiser-Meyer-Olkin) & \multicolumn{5}{|l|}{0.858} \\
\hline Bartlett's Test of Sphericity & \multicolumn{5}{|c|}{$2185.53, p=0.000$} \\
\hline Rate of Total Variansce Explanained (\%) & \multicolumn{5}{|c|}{$\% 68.28$} \\
\hline Cronbach Alpha & \multicolumn{5}{|c|}{0.9023} \\
\hline Item means (min -max) & \multicolumn{5}{|c|}{$3.30(2.18-4.22)$} \\
\hline Hotelling $\mathrm{T}$ test & \multicolumn{5}{|c|}{$376.68, p=0.000$} \\
\hline Corrected Item Total Corelation & \multicolumn{5}{|c|}{$0.32-0.65$} \\
\hline Nonadditivity & \multicolumn{5}{|c|}{$F=8.67, p=0.0032$} \\
\hline
\end{tabular}

Table 2: Factor analaysis of job satisfaction of garbage workers (26 questions, $\mathrm{N}=185$ ). 


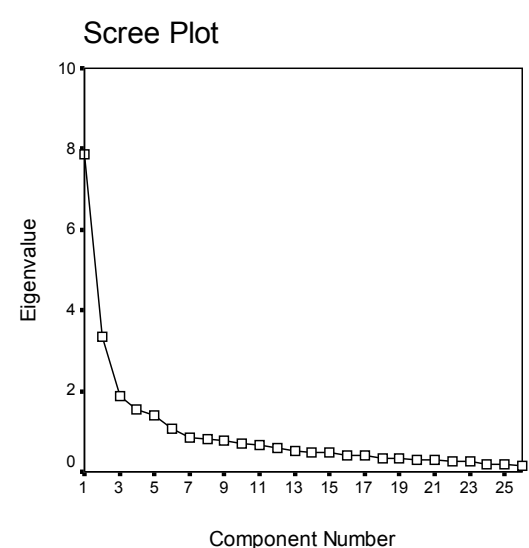

Figure 1: The screen plot of the extracted variables of JDI.

sufficient to determine $68.4 \%$ of job satisfaction. The screen plot of the extracted variables was showed in Figure 1. Five components which are eigenvalue were higher than one was evaluated according to this figure. Initial Eigenvalue of first component (variance rate of first factor) was $30.91 \%$ and rotation sum of squared loading of this component was $16.22 \%$. Initial Eigenvalues of second component was $14.16 \%$ and rotation sum of squared loading of this component was $15.24 \%$. First component included following items; management's respectful and fair treatment to workers, support and advicement to workers, recognizing workers' hard work by management, effective and efficient control, giving responsibility to workers, job satisfaction in general (26.item) (Table 2). Second component included 5 items related to "coworkers" from fourth facet. Third, fourth and fifth components had "pay", "promotion" and "work itself" items respectively.

The reliability of the JDI questionnaire was obtained by Cronbach's alpha coefficient $(\mathrm{a}=0.90)$. Item mean was $3.30(2.18-4.22)$ and corrected item total correlation was between 0.32- 0.65. Questions of our JDI was nonadditivity $(\mathrm{F}=8.67, \mathrm{p}<0.05)$ and means of questions was not equal (Hotelling $\mathrm{T}=376.68, \mathrm{p}<0.05)$.

Results of mean, standart deviation and median values of JDI and WHOQOL-BREF-TR and correlations between facets of JDI and domains of WHOQOL-BREF-TR

The mean score of first facet of JDI that was about supervision was $3.50 \pm 0.89$. The mean scores of other facets were as followings; the work itself $2.81 \pm 0.56$, pay $2.55 \pm 1.10$, coworkers $3.91 \pm 0.95$, promotions and promotion opportunities $3.07 \pm 0.75$. The mean and standard deviation of the last question about general job satisfaction was 3.46 \pm 1.39 .

The mean score of Physical domain of WHOQOL-BREF-TR was 13.32 \pm 2.68 , mean score of Psychological domain was $14.00 \pm 03.12$, mean score of Social domain was $14.59 \pm 3.76$ and mean score of Environment-TR domain was $11.86 \pm 2.65$. Besides their medians respectively were; $13.71,14.00,14.66$ and 12.00 .

The correlations between four facets of JDI (supervision, work itself, pay, coworkers) and all domains of WHOQOL-BREF-TR (physical, psychological, social and environment TR) were found $(\mathrm{p}<0.001-0.01)$ (Table 3). Additionally between question of general job satisfaction and all domains of WHOQOL-BREF-TR was found too $(\mathrm{p}<0.001)$. Besides, correlation was found between facet of promotions/promotion opportunities of JDI and environment domain only $(\mathrm{p}<0.05)$. No other correlations were found between age or work duration and all domains of WHOQOL-BREF-TR ( $\mathrm{p}>0.05)$.

\section{Results of relationships between domains of WHOQOL- BREF-TR and sociodemographic factors}

We observed significant statistical differences in between two opposite groups - people who have a chronical disease and those who don't, people who have hobbies and those who don't, people who go to movies, theaters or sport events and those who don't, people who like to visit relatives and friends and those who don't regarding to WHOQOL-BREF-TR average scoring associated with sociodemographic factors. $(\mathrm{p}<0.05)$ (Table 4). For instance people

\begin{tabular}{|c|c|c|c|c|c|}
\hline & Physical Domain & Psychological Domain & Social Domain & Environment Domain & $\begin{array}{l}\text { Environment Domain } \\
\text { TR }\end{array}$ \\
\hline Age & $\begin{array}{l}r=-0.028 \\
p=0.702\end{array}$ & $\begin{array}{l}r=-0.086 \\
p=0.244\end{array}$ & $\begin{array}{l}r=-0.078 \\
p=0.290\end{array}$ & $\begin{array}{l}r=-0.051 \\
p=0.487\end{array}$ & $\begin{array}{l}r=-0.055 \\
p=0.454\end{array}$ \\
\hline Duration working of this job & $\begin{array}{l}r=0.077 \\
p=0.299\end{array}$ & $\begin{array}{l}r=0.014 \\
p=0.854\end{array}$ & $\begin{array}{l}r=0.036 \\
p=0.623\end{array}$ & $\begin{array}{l}r=0.091 \\
p=0.219\end{array}$ & $\begin{array}{l}r=0.071 \\
p=0.335\end{array}$ \\
\hline Facet of supervision & $\begin{array}{l}r=0.521 \\
p<0.001\end{array}$ & $\begin{array}{l}r=0.469 \\
p<0.001\end{array}$ & $\begin{array}{l}r=0.431 \\
p<0.001\end{array}$ & $\begin{array}{l}r=0.453 \\
p<0.001\end{array}$ & $\begin{array}{l}r=0.457 \\
p<0.001\end{array}$ \\
\hline Facet of work itself & $\begin{array}{l}r=0.246 \\
p<0.001\end{array}$ & $\begin{array}{l}r=0.258 \\
p<0.001\end{array}$ & $\begin{array}{l}r=0.350 \\
p<0.001\end{array}$ & $\begin{array}{l}r=0.192 \\
p<0.01\end{array}$ & $\begin{array}{l}r=0.200 \\
p<0.01\end{array}$ \\
\hline Facet of pay & $\begin{array}{l}r=0.356 \\
p<0.001\end{array}$ & $\begin{array}{l}r=0.258 \\
p<0.001\end{array}$ & $\begin{array}{l}r=0.309 \\
p<0.001\end{array}$ & $\begin{array}{l}r=0.574 \\
p<0.001\end{array}$ & $\begin{array}{l}r=0.558 \\
p<0.001\end{array}$ \\
\hline Facet of coworkers & $\begin{array}{l}r=0.311 \\
p<0.001\end{array}$ & $\begin{array}{l}r=0.221 \\
p<0.01\end{array}$ & $\begin{array}{l}r=0.327 \\
p<0.001\end{array}$ & $\begin{array}{l}r=0.265 \\
p<0.001\end{array}$ & $\begin{array}{l}r=0.258 \\
p<0.001\end{array}$ \\
\hline $\begin{array}{l}\text { Facet of promotions \& promotion } \\
\text { opportunities }\end{array}$ & $\begin{array}{l}r=-0.071 \\
p=0.340\end{array}$ & $\begin{array}{l}r=0.035 \\
p=0.638\end{array}$ & $\begin{array}{l}r=-0.030 \\
p=0.682\end{array}$ & $\begin{array}{l}r=-0.148 \\
p<0.05\end{array}$ & $\begin{array}{l}r=-0.134 \\
p=0.068\end{array}$ \\
\hline General job satisfaction ( $26^{\text {th }}$ question) & $\begin{array}{l}r=0.353 \\
p<0.001\end{array}$ & $\begin{array}{l}r=0.387 \\
p<0.001\end{array}$ & $\begin{array}{l}r=0.363 \\
p<0.001\end{array}$ & $\begin{array}{l}r=0.448 \\
p<0.001\end{array}$ & $\begin{array}{l}r=0.442 \\
p<0.001\end{array}$ \\
\hline
\end{tabular}

Table 3: The correlations between facets of JDI and domains of WHOQOL-BREF-TR. 
Citation: Savas N, Nazlican E, Turhan E (2012) Job Satisfaction and Sociodemographic Factors Affectıng Garbage Workers' Quality of Lıfe in Antakya (Antioch), Turkey. Primary Health Care 2:110. doi:10.4172/2167-1079.1000110

Page 5 of 8

who don't have chronical disease and people who have hobbies scored higher than their opposite groups.

\section{Modality of risk in garbage worker's QOL with backward logistic regression analysis}

First, means of WHOQOL-BREF-TR domain scores of the workers were divided by two, and transformed into dicotom data. Then dicotom WHOQOL-BREF-TR scores - dependent variables - were processed with Backword Logistic regression analysis in order to find risk factors in workers' QOL. JDI facet values associated with WHOQOL-BREFTR, and sociodemographic factors related to WHOQOL-BREF-TR were used as independent variables. Table 5 shows output of logistic regression model. According to table data, "Provision" section created risk in physical, psychological, social and environmental domains, "Pay" section created in social and environmental domains, "Work itself" created in social domain only, "coworker" created risk in physical domain and "General Job Satisfaction" created risk in psychological and environmental domains (Question 26).
Odds Ratios calculated in Logistic Regression showed us, 1 point reduction in "Provision" would be equivalent to 1.18 times point reduction in physical domain, 1.16 times point reduction in psychological domain, 1.09 times point reduction in social domain and 1.1 times point reduction in environmental-TR domain. Comparing to people who have hobbies, those who don't would have 0.29 times risk of getting lower score than average (14.00) in psychological domain, and 0.46 times risk of getting lower score than average (11.50) in environmental domain.

\section{Discussion}

According to Heller, job satisfaction correlates strongly with life satisfaction [22]. Job satisfaction should not be studied alone, rather researchers should examine it within broader context of emotional lives of employees [9]. This research took the same approach by studying job satisfaction along with work related, socio-demographic factors and quality of life as well as emotional lives of employees. By our JDI's findings, $68.4 \%$ of job satisfaction includes supervision-

\begin{tabular}{|c|c|c|c|c|c|}
\hline \multirow[b]{2}{*}{ Sociodemographic Factors } & Physical Domain & Psychological Domain & Social Domain & Environment Domain & $\begin{array}{l}\text { Environment Domain- } \\
\text { TR }\end{array}$ \\
\hline & $\begin{array}{l}\mathrm{p}^{*} \\
\text { mean } \pm s d\end{array}$ & $\begin{array}{l}\mathrm{p}^{*} \\
\text { mean } \pm s d\end{array}$ & $\begin{array}{l}\mathrm{p}^{*} \\
\text { mean } \pm s d\end{array}$ & $\begin{array}{l}\mathrm{p}^{*} \\
\text { mean } \pm s d\end{array}$ & $\begin{array}{l}\mathrm{p}^{*} \\
\text { mean } \pm s d\end{array}$ \\
\hline $\begin{array}{l}\text { Education } \\
\text { None /Primary school } \\
\text { Other schools }\end{array}$ & $\begin{array}{l}p>0.05 \\
13.5 \pm 2.8 \\
13.3 \pm 2.6\end{array}$ & $\begin{array}{l}p>0.05 \\
14.3 \pm 3.0 \\
13.9 \pm 3.1\end{array}$ & $\begin{array}{l}p>0.05 \\
14.1 \pm 3.9 \\
14.6 \pm 3.7\end{array}$ & $\begin{array}{l}p>0.05 \\
11.6 \pm 3.3 \\
11.7 \pm 2.8\end{array}$ & $\begin{array}{l}p>0.05 \\
11.7 \pm 2.9 \\
11.8 \pm 2.6\end{array}$ \\
\hline $\begin{array}{l}\text { Marital status } \\
\text { Married } \\
\text { Other types }\end{array}$ & $\begin{array}{l}p>0.05 \\
13.2 \pm 2.6 \\
13.7 \pm 3.0\end{array}$ & $\begin{array}{l}p>0.05 \\
14.0 \pm 3.1 \\
13.7 \pm 3.6\end{array}$ & $\begin{array}{l}p>0.05 \\
14.6 \pm 3.7 \\
13.3 \pm 3.2\end{array}$ & $\begin{array}{l}p>0.05 \\
11.7 \pm 2.8 \\
12.1 \pm 3.3\end{array}$ & $\begin{array}{l}p>0.05 \\
11.8 \pm 2.6 \\
12.1 \pm 2.9\end{array}$ \\
\hline $\begin{array}{l}\text { Occupation of wife } \\
\text { Housewife } \\
\text { Other jobs }\end{array}$ & $\begin{array}{l}p>0.05 \\
13.2 \pm 2.6 \\
13.5 \pm 3.3\end{array}$ & $\begin{array}{l}p>0.05 \\
14.0 \pm 3.1 \\
13.5 \pm 3.3\end{array}$ & $\begin{array}{l}p>0.05 \\
14.7 \pm 3.8 \\
13.5 \pm 3.2\end{array}$ & $\begin{array}{l}p>0.05 \\
11.7 \pm 2.8 \\
11.7 \pm 3.1\end{array}$ & $\begin{array}{l}p>0.05 \\
11.8 \pm 2.6 \\
11.9 \pm 2.8\end{array}$ \\
\hline $\begin{array}{l}\text { Staying in house for rent } \\
\text { Absent } \\
\text { Present }\end{array}$ & $\begin{array}{l}p>0.05 \\
13.3 \pm 3.0 \\
13.2 \pm 2.4\end{array}$ & $\begin{array}{l}p>0.05 \\
14.4 \pm 3.1 \\
13.7 \pm 3.0\end{array}$ & $\begin{array}{l}p>0.05 \\
14.9 \pm 3.9 \\
14.3 \pm 3.6\end{array}$ & $\begin{array}{l}p>0.05 \\
11.7 \pm 2.8 \\
11.7 \pm 2.9\end{array}$ & $\begin{array}{l}p>0.05 \\
11.9 \pm 2.5 \\
11.8 \pm 2.7\end{array}$ \\
\hline $\begin{array}{l}\text { Family type } \\
\text { Nuclear family } \\
\text { Other types }\end{array}$ & $\begin{array}{l}p>0.05 \\
13.3 \pm 2.6 \\
13.4 \pm 2.8\end{array}$ & $\begin{array}{l}p>0.05 \\
13.9 \pm 3.0 \\
14.2 \pm 3.5\end{array}$ & $\begin{array}{l}p>0.05 \\
14.6 \pm 3.7 \\
14.2 \pm 3.7\end{array}$ & $\begin{array}{l}p>0.05 \\
11.6 \pm 2.8 \\
12.2 \pm 3.1\end{array}$ & $\begin{array}{l}p>0.05 \\
11.8 \pm 2.5 \\
12.1 \pm 2.9\end{array}$ \\
\hline $\begin{array}{l}\text { Working children } \\
\text { Absent } \\
\text { Present }\end{array}$ & $\begin{array}{l}p>0.05 \\
13.3 \pm 2.5 \\
13.4 \pm 3.5\end{array}$ & $\begin{array}{l}p>0.05 \\
14.0 \pm 3.0 \\
13.3 \pm 3.4\end{array}$ & $\begin{array}{l}p>0.05 \\
14.6 \pm 3.7 \\
14.0 \pm 3.6\end{array}$ & $\begin{array}{l}p>0.05 \\
11.7 \pm 2.7 \\
11.4 \pm 3.8\end{array}$ & $\begin{array}{l}p>0.05 \\
11.9 \pm 2.5 \\
11.4 \pm 3.3\end{array}$ \\
\hline $\begin{array}{l}\text { Disabled/patient in the house } \\
\text { Absent } \\
\text { Present }\end{array}$ & $\begin{array}{l}p>0.05 \\
13.3 \pm 2.6 \\
13.1 \pm 2.7\end{array}$ & $\begin{array}{l}p>0.05 \\
13.9 \pm 3.2 \\
14.1 \pm 2.4\end{array}$ & $\begin{array}{l}p>0.05 \\
14.6 \pm 3.7 \\
14.3 \pm 3.8\end{array}$ & $\begin{array}{l}p>0.05 \\
11.7 \pm 2.9 \\
11.8 \pm 3.0\end{array}$ & $\begin{array}{l}p>0.05 \\
11.8 \pm 2.6 \\
11.6 \pm 2.6\end{array}$ \\
\hline $\begin{array}{c}\text { Currently ill } \\
\text { Absent } \\
\text { Present }\end{array}$ & $\begin{array}{l}p=0.05 \\
12.5 \pm 3.0 \\
13.5 \pm 2.5\end{array}$ & $\begin{array}{l}P=0.006 \\
12.7 \pm 3.3 \\
14.3 \pm 2.9\end{array}$ & $\begin{array}{l}P=0.001 \\
12.7 \pm 4.0 \\
15.0 \pm 3.5\end{array}$ & $\begin{array}{l}P=0.05 \\
10.8 \pm 3.2 \\
11.9 \pm 2.7\end{array}$ & $\begin{array}{l}P=0.03 \\
11.0 \pm 2.8 \\
12.0 \pm 2.5\end{array}$ \\
\hline $\begin{array}{l}\text { Working hour } \\
\text { 07:00-17:00 or } 11: 00-21: 00 \\
03: 00-11: 00 \text { or } 22: 00-06: 00\end{array}$ & $\begin{array}{l}p>0.05 \\
13.3 \pm 2.4 \\
13.3 \pm 2.8\end{array}$ & $\begin{array}{l}p>0.05 \\
13.7 \pm 2.6 \\
14.2 \pm 3.5\end{array}$ & $\begin{array}{l}p>0.05 \\
14.1 \pm 3.4 \\
15.0 \pm 4.0\end{array}$ & $\begin{array}{l}p>0.05 \\
11.7 \pm 2.7 \\
11.7 \pm 3.1\end{array}$ & $\begin{array}{l}p>0.05 \\
11.8 \pm 2.4 \\
11.8 \pm 2.8\end{array}$ \\
\hline $\begin{array}{l}\text { Part time job } \\
\text { Yes } \\
\text { Sometimes or No }\end{array}$ & $\begin{array}{l}p>0.05 \\
13.2 \pm 2.8 \\
13.8 \pm 1.8\end{array}$ & $\begin{array}{l}p>0.05 \\
13.9 \pm 3.2 \\
14.1 \pm 2.6\end{array}$ & $\begin{array}{l}p>0.05 \\
14.6 \pm 3.8 \\
14.5 \pm 3.2\end{array}$ & $\begin{array}{l}p>0.05 \\
11.6 \pm 2.8 \\
12.2 \pm 3.0\end{array}$ & $\begin{array}{l}p>0.05 \\
11.7 \pm 2.6 \\
12.4 \pm 2.7\end{array}$ \\
\hline $\begin{array}{l}\text { Spending time with hobbies } \\
\text { Yes } \\
\text { Sometimes or No }\end{array}$ & $\begin{array}{l}P=0.001 \\
14.0 \pm 2.3 \\
12.7 \pm 2.7\end{array}$ & $\begin{array}{l}\mathbf{p}<0.001 \\
15.1 \pm 2.8 \\
13.1 \pm 3.0\end{array}$ & $\begin{array}{l}P=0.04 \\
15.2 \pm 3.7 \\
14.1 \pm 3.7\end{array}$ & $\begin{array}{l}P=0.009 \\
12.3 \pm 2.9 \\
11.2 \pm 2.7\end{array}$ & $\begin{array}{l}\mathbf{P}=\mathbf{0 . 0 1} \\
12.4 \pm 2.6 \\
11.4 \pm 2.6\end{array}$ \\
\hline $\begin{array}{l}\text { Going out to movies/ theaters/ ball } \\
\text { games } \\
\text { Yes } \\
\text { Sometimes or No }\end{array}$ & $\begin{array}{l}p=0.002 \\
14.1 \pm 2.2 \\
12.9 \pm 2.7\end{array}$ & $\begin{array}{l}p=0.037 \\
14.7 \pm 3.07 \\
13.6 \pm 3.1\end{array}$ & $\begin{array}{l}p>0.05 \\
15.0 \pm 3.2 \\
14.3 \pm 3.9\end{array}$ & $\begin{array}{l}p=0.004 \\
12.6 \pm 2.6 \\
11.3 \pm 2.9\end{array}$ & $\begin{array}{l}p=0.003 \\
12.6 \pm 2.3 \\
11.4 \pm 2.7\end{array}$ \\
\hline $\begin{array}{l}\text { Visiting friends/relatives } \\
\text { Yes } \\
\text { Sometimes or No }\end{array}$ & $\begin{array}{l}\mathbf{p}<0.001 \\
13.6 \pm 2.4 \\
11.4 \pm 3.1\end{array}$ & $\begin{array}{l}p=0.001 \\
14.3 \pm 2.9 \\
12.1 \pm 3.6\end{array}$ & $\begin{array}{l}P=0.004 \\
14.9 \pm 3.6 \\
12.6 \pm 4.2\end{array}$ & $\begin{array}{l}p=0.002 \\
12.0 \pm 2.7 \\
10.0 \pm 3.3\end{array}$ & $\begin{array}{l}p=0.004 \\
12.0 \pm 2.5 \\
10.4 \pm 3.0\end{array}$ \\
\hline
\end{tabular}

Student $T$ test

Table 4: The relationships sociodemographic factors and domains of WHOQOL-BREF 


\begin{tabular}{|c|c|c|c|c|c|c|c|c|c|c|}
\hline & \multicolumn{2}{|c|}{$\begin{array}{l}\text { Physical Domain } \\
<\text { median - } \geq \text { median }\end{array}$} & \multicolumn{2}{|c|}{$\begin{array}{l}\text { Psychological Domain } \\
<\text { median - } \geq \text { median }\end{array}$} & \multicolumn{2}{|c|}{$\begin{array}{l}\text { Social Domain } \\
<\text { median - } \geq \text { median }\end{array}$} & \multicolumn{2}{|c|}{$\begin{array}{l}\text { Environment Domain } \\
<\text { median - } \geq \text { median }\end{array}$} & \multicolumn{2}{|c|}{$\begin{array}{l}\text { Environment Domain } \\
\text { TR } \\
<\text { median - } \geq \text { median }\end{array}$} \\
\hline & OR & p & OR & p & OR & $\mathbf{p}$ & OR & p & OR & p \\
\hline Constant & & $<0.001$ & & $<0.05$ & & $<0.001$ & & $<0.001$ & & $<0.001$ \\
\hline \multicolumn{11}{|l|}{ JDI facets } \\
\hline Supervision & 1.18 & $<0.001$ & 1.16 & 0.001 & 1.09 & 0.04 & 1.21 & $<0.001$ & 1.10 & 0.04 \\
\hline The work itself & & NS & & NS & 1.21 & 0.001 & & NS & & NS \\
\hline Pay & & NS & & NS & 1.09 & 0.009 & 1.23 & $<0.001$ & 1.16 & $<0.001$ \\
\hline Coworkers & 1.11 & $<0.01$ & & NS & & NS & & NS & & NS \\
\hline $\begin{array}{l}\text { Promotions \& promotion } \\
\text { opportunities }\end{array}$ & & NS & & NS & & NS & & NS & & NS \\
\hline $\begin{array}{l}\text { Currently ill } \\
\text { Absent } \\
\text { Present }\end{array}$ & & NS & & NS & & NS & & NS & & NS \\
\hline $\begin{array}{l}\text { General job satisfaction } \\
\left(26^{\text {th }} \text { question }\right)\end{array}$ & & NS & & NS & & NS & & NS & 1.42 & 0.02 \\
\hline $\begin{array}{l}\text { Spending time with hobb } \\
\text { No } \\
\text { Yes or sometimes }\end{array}$ & & NS & 0.29 & $<0.001$ & & NS & 0.46 & 0.04 & & NS \\
\hline $\begin{array}{l}\text { Going out to movies/thea } \\
\text { ball games } \\
\text { No } \\
\text { Yes or sometimes }\end{array}$ & & NS & & NS & & NS & & NS & & NS \\
\hline $\begin{array}{l}\text { Visiting friends/relatives } \\
\text { No } \\
\text { Yes or sometimes }\end{array}$ & & NS & & NS & & NS & & NS & & NS \\
\hline
\end{tabular}

*Backward LR

NS: no significant

Table 5: Analysis of sociodemographic properties and scores of JDI facets with WHOQOL-BREF domains using logistic regression ${ }^{\star}$ model.

general job satisfaction, coworkers, pay, promotions, and work itself. Supervision component is the first factor on the list as our study found. Another finding by Bush was that it's essential for management to help workers gain more skills, support personnel, and help them keep good relations with public [23]. According to Herzberg's double factor theory, business and management policies, technical surveillance, poor working conditions and relations among workers can cause negative behaviors [24]. Another research says; supervisor support and workfamily culture were positively related to job satisfaction and affective commitment [25]. On the other hand, job characteristic or job itself was positively related to all measures of job outcome [25]. However, we found that work itself was the least important component of job satisfaction of the garbage workers. The result of Mount and Bartlett's research suggested that job satisfaction has the least important relationship with pay component [26]. Also we found that pay component ranked after supervision and co-workers components.

According to average JDI components points from highest to lowest, factors that affect job satisfaction can be sorted as coworkers, supervision, general job satisfaction, promotions/promotion opportunities, the work itself and pay. As seen from this order, garbage workers are satisfied with "coworkers" the most, "salary" the least. But in the study, none of these factors got a mean score of 4 or over, on the scale of 5. According to the result of pay component; we may explain that our research group has one of the lowest salaries for living condition of their parents in Turkey. Besides their income are very little in compare to their job which is exhausting and unhygienic.

"Coworkers" component showed us, workers were in favor of collectivisms. The findings of Yetim et al. [27] study revealed that collectivism, paternalism, and power distance predicted employee job satisfaction significantly. In addition, the extrinsic, intrinsic, and general job satisfaction have a significant effect on normative commitment and affective commitment [28]. Income level affects affective commitment indirectly [28]. Institutions in Turkey usually focus on organizational commitment and job satisfaction correlation among the employees in different regions of Turkey within one organization [28].

Considering average points in WHOQOL-BREF-TR domains, job satisfaction components can be ordered by their significances as follows; social, psychological, physical and environmental. Another study also shows, the sample of 698 male automotive assembly workers in Malaysia list them in the following order; social, physical, psychological and environmental [19]. Among WHOQOL-BREF-TR questions that determined social domain points are; "How much are you dissatisfied in your relationships with other individuals?", "How much are you dissatisfied in your sexual life?", "How much are you dissatisfied of your friends' support?". Results from researches done in both Malaysia and Turkey concluded that friendship among workers, working both indoor and outdoor increases quality of social life. The garbage worker's quality of life about social area can be better than other areas. Because garbage workers according to the role they perform in a community, the social area's quality of life better than unhygienic environment. Also, their social life areas is not their working areas. Perhaps they think that the community feel sorry to them and underrate them while they are working, so their psychological area's quality of life is affected badly.

Although, according to different study; instead of WHOQOLBREF, SF-36 was used to examine 107 workers including, 33 exposed to vibration, 27 to various hazards mostly chemical, and 47 individuals without any exposure [29]. The latter group examinees had lower life quality in Pain scale, Social functioning, Vitality, General Health scale. In all the groups, psychical health was decreased [29].

The correlations between four facets of JDI (supervision, work itself, pay, coworkers) and all domains of WHOQOL-BREF-TR 
(physical, psychological, social and environment TR) were found in this study. Additionally the correlations between question of general job satisfaction and all domains of WHOQOL-BREF-TR were found too.

We did not find a correlation between physical domain and work duration during entire years of our study. But, Nordlund's case-control study done in 1989-1997, there was a increasing trend for prevalence of musculoskeletal symptoms, as well as decreasing health status as rated in the SF-36. Duration of our study took $4.94 \pm 4.17$ years long depending on different research groups, while Nordlund et al. [30] studies took much longer. Additionally we did not find a correlation between all domain and their ages too. But, the study from Almeida et al. [31] in garbage collectors in Brazil showed that the youngest garbage collectors presented a lower degree of life satisfaction.

Based on the analysis of logistic regression, one of the workers had a lower point than the average for quality of life in four different domains which are social, psychological, physical and environmentTR. Job satisfaction facets that contributes to risk in quality of life can be summarized as follows. "Supervision" affects on all four domains, "Work itself" affects in social domain, "co-workers" in physical domain, while pay affects in both social and environmental domains.

Edimansyah et al. [32] supported that the Karasek's job strain and iso-strain models which research on the association between job stress and health. The Karasek's models are important determinants of self-perceived QOL among male automotive workers in Malaysia [32]. Particularly supervision facet may cause work stress, because it contributes to risk in decreasing quality of life. We could have probably seen it, should we studied work stress.

A strong correlation was found between JDI's coworkers group whose average point is the highest and WHOQOL-BREF-TR social group with the same characteristic. But logistic regression model, we concluded that low scoring in "coworkers" factor has no increasing risk effect in getting lower score than the average in social area. Not having a visitor (friend/relative) time had no effect either. Low scorings in "Supervision", "work itself" and "pay" are found to have increasing risk effects on getting low scores in social area.

How "work itself" causes a risk in quality of life in social area. "Work itself" section has the second worst scoring after "pay" section that affects job satisfaction. "Social" area had the highest score in quality of life. According to JDI's study about "Work itself" section, worker's disrespect to his/her work, evaluating his work as too tiring, believing that he/she has no job security can cause risk in having poor quality of life in social area. It could also cause to loose self confidence, sexual life and relationships with others.

We expected that dissatisfaction on "pay" causes poor quality of life in environmental area. Among the questions we asked to workers were: "Do you have enough money to buy what you need?", "Is your physical environment healthy?", "How much are you dissatisfied in your living conditions of your house?" These questions are directly related to pay. We expected that "having no hobby" contributed to poor quality of life in environmental area. Because, "Do you have time for your hobbies?" is one of the questions that effect quality of life in environmental area.

In our study, dissatisfaction in "Coworkers" section had a risk factor to quality of life in physical area. As a result of this, having no responsibilities and help among coworkers, being around lazy coworkers effects the worker physically, causing poor quality of life in physical area.

The result of Edimansyah's study, "Relationship of psychosocial work factors and health-related quality of life in male automotive assembly workers in Malaysia" that Multiple linear regression analysis indicated that created skill was positively associated with physical health and psychological domains; whilst, skill discretion was positively associated with social relationship and environment domains. Social support was positively associated with physical health and environment domains; whilst, co-worker support was positively associated with psychological and social relationship domains [33].

\section{Conclusion and Recommendations}

As a conclusion, factors affecting job satisfaction among Antakya Garbage Workers are management/supervision, general job satisfaction, coworkers, pay, promotion and work itself. Considering four areas of social, psychological, physical and environment-TR, each factor carries a risk in different areas causing poor quality of life.

- Dissatisfaction in supervision/management had effects on all of the four areas.

- Pay dissatisfaction had effects on psychological, and environment-TR areas.

- Dissatisfaction to coworkers had effects on physical and physical areas.

- Having no hobbies had effects on psychological and environment-TR areas.

Improving supervision, pay, and friendship within coworkers and supporting hobbies are main contributing factors to increase quality of life among workers.

\section{References}

1. WHOQOL Group (1995) The World Health Organization Quality of Life assessment (WHOQOL): position paper from the World Health Organization. Soc Sci Med 41: 1403-1409.

2. Locke EA (1969) What is Job Satisfaction. Organizational Behaviour and Human Performance 4: 309-336.

3. Locke EA, Whiting RJ (1974) Sources of satisfaction and dissatisfaction among solid waste management employees. Journal of Applied Psychology 59: 145156 .

4. Locke EA (1976) The Nature and Causes of Job Satisfaction. Handbook of Industrial and Organizational Psychology, Rand McNally College Publishing Company, Chicago, 1297-1349

5. Ozgen H, Ozturk A, Yalcin A (2002) Human Resources Management. Nobe Publishing Company, Adana, Turkey.

6. Lerner DJ, Levine S, Malspeis S, D'Agostino RB (1994) Job strain and healthrelated quality of life in a national sample. Am J Public Health 84: 1580-1585.

7. Nasermoaddeli A, Sekine M, Hamanishi S, Kagamimori S (2003) Associations between sense of coherence and psychological work characteristics with changes in quality of life in Japanese civil servants: a 1-year follow-up study. Ind Health 41: 236-241.

8. Kudielka BM, Hanebuth D, Von Kanel R, Gander ML, Grande G, et al. (2005) Health-related quality of life measured by the SF12 in working populations: associations with psychosocial work characteristics. J Occup Health Psychol 10: $429-440$.

9. Heller D, Judge TA, Watson D (2002) The confounding role of personality and trait affectivity in the relationship between job and life satisfaction. Journal of Organizational Behavior 23: 815-835. 
Citation: Savas N, Nazlican E, Turhan E (2012) Job Satisfaction and Sociodemographic Factors Affectıng Garbage Workers' Quality of Lıfe in Antakya (Antioch), Turkey. Primary Health Care 2:110. doi:10.4172/2167-1079.1000110

10. Herzberg F (1966) Work and the Nature of Man, Cleveland: World Publishing Co.

11. http://tuikapp.tuik.gov.tr/adnksdagitapp/adnks.zul.

12. Smith, PC, Kendall LM, Hulin CL (1969) The measurement of satisfaction in work and retirement. Rand-McNally, Chicago.

13. Kerr BA (1985) Review of the Job Descriptive Index. Ninth Mental Measurements Yearbook, University of Nebraska Press, Lincoln, NE 1: 754-755.

14. Ergin C (1997) Job Description of Job Satisfaction as a Measure Scale: Adaptation, Validity and Reliability Study (in Turkish). Turkish Journal of Psychology 12: 25-36.

15. WHOQOL Group (1998) Development of the World Health Organization WHOQOL-BREF quality of life assessment. Psychol Med 28: 551-558.

16. WHOQOL Group (1998) The World Health Organization quality of life assessment (WHOQOL): Development and general psychometric properties. Social Science and Medicine 46: 1569-1585.

17. Carr AJ, Higginson IJ (2001) Are quality of life measures patient centred. BMJ 322: $1357-1360$

18. Eser E, Fidaner H, Fidaner C (1999) WHOQOL-BREF TR: a suitable instrument for the assessment of quality of life for use in the health care settings in Turkey (abstract no 433). Quality of Life Research 8: 647.

19. Rusli BN, Edimansyah BA, Naing L (2008) Working conditions, self-erceived stress, anxiety, depression and quality of life: A structural equation modelling approach. BMC Public Health 8: 48.

20. Pallant JF (2000) Development and validation of a scale to measure perceived control of internel states. J Pers Assess 75: 308-337.

21. Kaiser HF (1960) The application of electronic computers to factor analysis. Education and pyschological measurement 20: 141-151.

22. Judge TA, Larsen RJ (2001) Dispositional source of job satisfaction: a review and theoretical extension. Organizational Behavior and Human Decision Processes 86: 67-98.

23. Bush J (2002) 12 Tips for Increasing Your Job Satisfaction. Fam Pract Manag

\section{9: 70 .}

24. Bernard L. Hinton (1968) An empirical investigation of the Herzberg methodology and two-factor theory. Organizational Behavior \& Human Performance 3: 286309.

25. Baral R, Bhargava S (2010) Work-family enrichment as a mediator between organizational interventions for work-life balance and job outcomes. Journal of Managerial Psychology 25: 274-300.

26. Mount, DJ, Bartlett AL (2002) Development of a Job Satsfaction Factor Mode for The Lodging Industry, Journal of Human Resources in Hospitality \& Tourism 1: 17-39.

27. Yetim N, Yetim U (2006) The cultural orientations of entrepreneurs and employees' job satisfaction: the Turkish small and medium sized enterprises (SMEs) case. Social Indicator Research 77: 257-286.

28. Gunlu E, Aksarayli M, Percin NS (2010) Job satisfaction and organizationa commitment of hotel managers in Turkey. International Journal of Contemporary Hospitality Management 22: 693.

29. Liubchenko PN, Sorokina EV, lan'shina EN (2009) Life quality of workers in machinery plants of Moscow region. Med Tr Prom Ekol 2: 38-41.

30. Nordlund A, Ekberg K (2004) Self reported musculoskeletal symptoms in the neck/shoulders and/or arms and general health (SF-36): eight year follow up of a case-control study. Occupational and Environmental Medicine 61: 11.

31. Almeida JR, Elias ET, Magalhães MA, Vieira AJ (2009) Age effect on the life quality and health of garbage collectors of an association in Governador Valadares, Minas Gerais State, Brazil. Cien Saude Colet 14: 2169-2179.

32. Edimansyah BA, Rusli BN, Naing L (2007) Associations between job strain, ISO-straın and self-perceived quality of life in automotive assembly workers: a structural equation modeling approach. The Malaysian Journal of Medical Sciences 14: 267.

33. Edimansyah BA, Rusli BN, Naing L, Mohamed Rusli BA, Winn T (2007) Relationship of psychosocial work factors and health-related quality of life in male automotive assembly workers in Malaysia. Ind Health 45: 437-448. 